\title{
A Comparative Study of Sentiment Analysis on Mask-Wearing Practices during the COVID-19 Pandemic
}

\author{
Bishrul Haq ${ }^{1}$, Ghulam Mujtaba ${ }^{1,2}$, Zahid Hussain Khand ${ }^{1}$, Javed Ahmed ${ }^{1,2}$, Zafar Ali ${ }^{1}$ \\ ${ }^{1}$ Department of Computer Science, Sukkur IBA University, Sukkur, Pakistan \\ ${ }^{2}$ Center of Excellence for Robotics, Artificial Intelligence, and Blockchain, Sukkur IBA University, Sukkur, Pakistan \\ ${ }^{*}$ Corresponding author:bishrulhaq. mscsf19@iba-suk.edu.pk
}

\section{Abstract}

COVID-19 has become one of the most highly orated subject matter in these days. Countries have taken many viable actions to prevent the spread of the virus directed by international recommendations, which led to many disputes concerning wearing a face mask as a preventive measure against the virus. This study aims to assess and compare the overall accuracy, macro precision, macro F-measure and macro recall of the different decision models towards the COVID-19 mask-wearing practices via sentiment analysis. Tweets are labeled and text pre-processing techniques are applied as stemming, normalization, tokenization, and stop-word removal. Subsequently, the tweets are transformed into master feature vectors by applying various feature extraction, feature representation, feature selection and word embedding techniques with five supervised machine learning decision models to predict maskwearing practices reinforced from Twitter tweets. Moreover, the highest macro F-measure and macro precision are found with feature extraction as hybrid-grams, feature representation as TF-IDF, feature selection as Chi-Squared Test, and highest macro recall with feature extraction as BOW, feature representation as TF-IDF, feature selection as ANOVA F-value. Hence, this study concludes that the Naive Bayes (NB) algorithm outperforms other decision models with master feature vectors applied. In addition, it also outperforms word embedding techniques.

Keywords-COVID-19 pandemic, feature engineering, machine learning, mask-wearing practices, natural language processing, sentiment analysis, supervised decision models.

\section{Introduction}

$\mathrm{T}$ HE novel Coronavirus, known as COVID-19, is an emergency respiratory disease that was initially identified in Wuhan, China, on December 19. It is considered to be extremely contagious, and its main symptoms include fatigue, fever, dry cough, dyspnea, and myalgia [1]. It spreads by human to human virus transmission. Hence, distancing from a susceptible population is a better way to control the spread of the COVID-19 [2]. There are plenty of non-pharmaceutical approaches available in protecting from the virus. Hand washing and wearing face masks are ways to keep yourself protected from the virus [3].

Currently, there is no proper vaccine that has been identified for Covid-19. It is considered a severe illness that leads to many deaths and the healthcare systems

ISSN: 2523-0379 (Online), ISSN: 1605-8607 (Print)

DOI: 10.52584/QRJ.1802.17

This is an open access article published by Quaid-e-Awam University of Engineering Science $\&$ Technology, Nawabshah, Pakistan under CC BY 4.0 International License. being under pressure [4]. Relatively, wearing a face mask helps prevent the virus from transmitting the disease to another healthy person or prevents the illness by reducing the probability of infection. Wearing a face mask has been a controversial topic, and many had a variety of suggestions. However, On April 3, 2020, the CDC (Centers for Disease Control and Prevention) of the US has recommended the public to wear face masks [5]. Mask wearing practices have shown more success and have lessened the virus's threat in the past during the 2003 SARS epidemic [6]. Social media has gained popularity in recent years. It is regularly used among the elderly, teenagers, academics, politicians, and entrepreneurs, irrespective of age or gender. Twitter is one of the commonly used micro-blogging sites among users today. It confines users to tweet within 140 characters. Whereas it allows the users to use a variation of spellings, indecorous use of punctuation marks, slangs, and emojis [7]. This led many researchers to work on sentimental analysis. It is 
a part of natural language processing whose primary focus is on identifying and classifying sentiments or opinions in a textual format. Sentiments can be known as the emotions of service, brand, or campaign in social media platforms. It is a phenomenal way to elaborate the sentiments via comments or situations to understand the surrounding of the brand, service, or campaign [8]. Sentiment analysis has been widely applied in many studies due to the fact that many social media users have their freedom to express their opinions, thoughts, and suggestion for any viral or sensational issue [9]. Hence, it leads to identifying their impression concerning certain topics and current happenings. Understanding whether the given opinion falls into positive or negative sentiment is considered a difficult task. The given features applied in a sentence must contain strong adjectives to classify them. Moreover, sentiment analysis helps to determine the satisfaction of goods or services before they are purchased. Companies and corporations use this information to understand their products and selling patterns [10]. In sentiment analysis, supervised and unsupervised machine learning algorithms play a vital role. Hence, machine learning techniques are used to split the data into test and train. Train data is used to build a classifier to classify the sentiments of given data [10]. If the instances are given as labeled, it falls into the category of supervised learning [11].

The study presented in this paper aspires to achieve three main objectives. Firstly, to compare and contrast the differences in performance measures of five supervised machine learning decision models such as SVM (Support Vector Machine), NB (Naive Bayes), RF (Random Forest), KNN (K-Nearest Neighbour Algorithm), and MaxEnt (Maximum Entropy). Secondly, to evaluate the best feature value representation with feature extraction as BOW (Bag-of-words), $\mathrm{N}$-grams. Whereas, in $\mathrm{N}$-gram, three techniques are compared, i.e., bigram $(\mathrm{N}=2)$, trigram $(\mathrm{N}=3)$, and hybrid-grams (BOW + bigram + trigram). In feature representation, Binary Frequency (BR), TF (Term Frequency), TF-IDF (Term Frequency - Inverse Document Frequency), and CountVectorizer are used to convert the text into vectors. Moreover, in feature selection, three techniques are used, i.e. Chi-squared test, information gain, and Anova F-value. Thirdly, feature value representation is compared via Word embedding techniques applying GloVe and Word2Vec embeddings. Hence, the corpus is developed from Twitter tweets by eliminating retweets and tweet duplicates with pre-processing techniques by filtering unwanted symbols, special characters, null values, stop word removal, and correcting misspelled words. Hence, it is applied in the training model with 1200 tweets labeled as negative and positive, with 600 tweets per each class.

The rest of the paper is organized into the following sections. Related work is discussed in section 2. Section 3 discusses the implementation carried out in the research methodology. Section 4 includes the results. Section 5 is for the discussion of the study. Finally, the conclusion of the study is presented in section 6 .

\section{Related Work}

COVID-19 is a newly transpired disease that is known to be a new research area. However, fewer studies have been conducted in regards to sentiment analysis by the time of piloting this study. Moreover, there have been some studies on sentiment analysis with supervised machine learning algorithms, which to a certain extent, are related to our study.

In Anjaria and Guddeti [12], the authors use Twitter as the primary data collection. Whereas, it is a great platform that intends a quick and effective way in collecting the sentiments. Hence, the sentiment of a sentence is derived by the occurrence of the words which can be positive or negative based on the tone of the words [13]. The study applies several feature extraction techniques to extract the data such as unigram, bigram and (unigram + bigram) hybrid. Hence the results indicate SVM along with the hybrid approach gives a better accuracy compared to NB, MaxEnt, SVM and ANN. The study presents an overview of collecting the tweets, cleaning data and feature extraction to predict the outcome.

Sethi et. al. [14] conduct a study to analyze the emotions of twitter users via tweets related to COVID19. The proposed model is used to analyze the actual sentiments of the tweets. The dataset for the study is manually created via a twitter API with COVID19 and coronavirus hashtags. Moreover, the study uses MaxEnt, MNB (Multinomial Naive Bayes), decision tree, RF, XGBoost, SVM algorithms with N-gram representations as unigram, bigram and unigram + bigram + trigram. Hence, the study concludes that SVM and decision tree classifier performs better. Although, SVM is considered to be more stable during the experimental process.

Eshan and Hasan [15] pilot a study to detect abusive Bangali text using machine learning algorithms with unigram, bigram and trigram as feature extraction techniques based CountVectorizer and TF-IDF for feature representation. The study uses RF, SVM with Polynomial and Linear kernel, MNB as algorithms. The study finds SVM linear kernel with TF-IDF to 
perform better compared to CountVectorizer; albeit, the study does not apply any feature selection techniques.

Comparative research by Mujtaba et al. [16] finds the cause of death (COD) via the autopsy reports. Hence, the study uses unigram, bigram, trigram and hybrid-grams for feature extraction by representing the feature values using BR, TF and TF-IDF. Furthermore, Chi-squared test, information gain and Pearson correlation approaches are used. The study applies five popular decision models, i.e., SVM, J48, RF, NB, KNN to evaluate the performance of each classifier. This study states that SVM performs better than other decision models. Moreover, the study presents a complete methodological structure from data collection to evaluation metrics. Hence, the study confirms the classification accuracy based on several feature value representations.

Samuel et. al. [17] conduct a study on the fear sentiment progression over the peak time of COVID-19 in the United States. The study finds that classification accuracy of $91 \%$ for short tweets with the NB decision model and MaxEnt performs similarly well with an accuracy of $74 \%$ with short tweets and it also reveals that both models perform weaker in longer tweets. The study applies 4 decision models, i.e., NB, MaxEnt, Linear Regression and K-Nearest Neighbor; albeit the study does not apply any feature selection technique to select the best features from the document of words. Sharma and Daniels [18] conduct a study to execute sentiment analysis based on 2019 election twitter tweets with Word2Vec word embedding along with RF classification model. Moreover, it also improve the accuracy compared to other feature representation schemes, i.e., BOW and TF-IDF. Hence, the study proposes that Word2Vec embedding improves the quality of features, thus it signifies the importance on word embedding in sentiment analysis. However, to the best of our knowledge, there is no existing study related to mask-wearing practices during the Covid-19 pandemic using sentiment analysis.

\section{Methodology}

The methodology of the study emphasizes the scheme of analyzing the sentiments towards mask-wearing practices during the COVID-19 pandemic. Hence, the study is divided into nine main steps as illustrated in Figure 1. Each step carries certain tasks and it is discussed in the subsequent sections of the methodology. The study uses supervised machine learning decision models to classify with the training and test sets. We have taken $60 \%$ dataset for training and the remaining

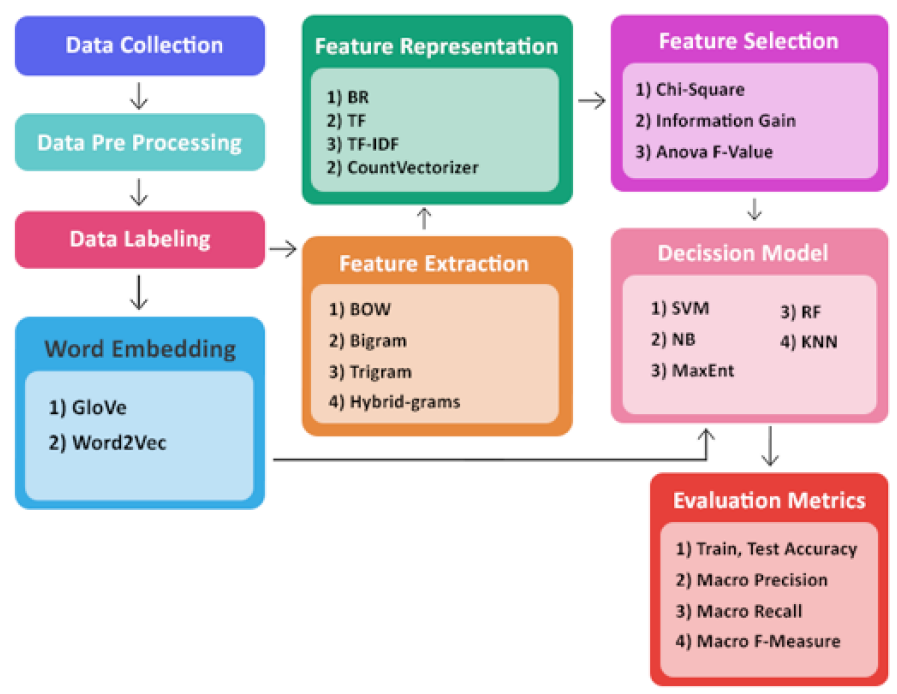

Fig. 1: Methodology of the Study

for testing. The sentiment for the instance is manually labeled with two categories which helps to create the prediction model. Whereas, the model is accountable to calculate the label for the unlabeled test set.

\subsection{Data Collection}

This study uses Twitter tweets as the primary data during Covid-19 pandemic which focuses on maskwearing practices. Whereas, tweets are collected using Twitter API with Python Tweepy Library [19]. We have created a Twitter developer account to extract the tweets via Python Tweepy Library. The gathered 5000 tweets contain a user ID, username, tweet text and tweet location of the tweet posted by the user. Tweets are gathered using the following three main criteria.

1) Tweets published during the COVID 19 pandemic are selected for the study.

2) Tweets are selected with relevant hashtags and search queries related to mask-wearing practices.

3) Tweets are extracted from Twitter API as extended, untruncated Tweet text.

\subsection{Data Pre-processing and Data Labeling}

The collected twitter tweets are then moved to the preprocessing phase. In this phase, some pre-processing techniques are applied to clean the data found in several retweets from different users. Hence, we keep one tweet and remove the duplicates. Then, unwanted characters, punctuation marks, numerical values are removed. Tweets are then converted into lowercase. Empty or null tweets are removed and misspelled 


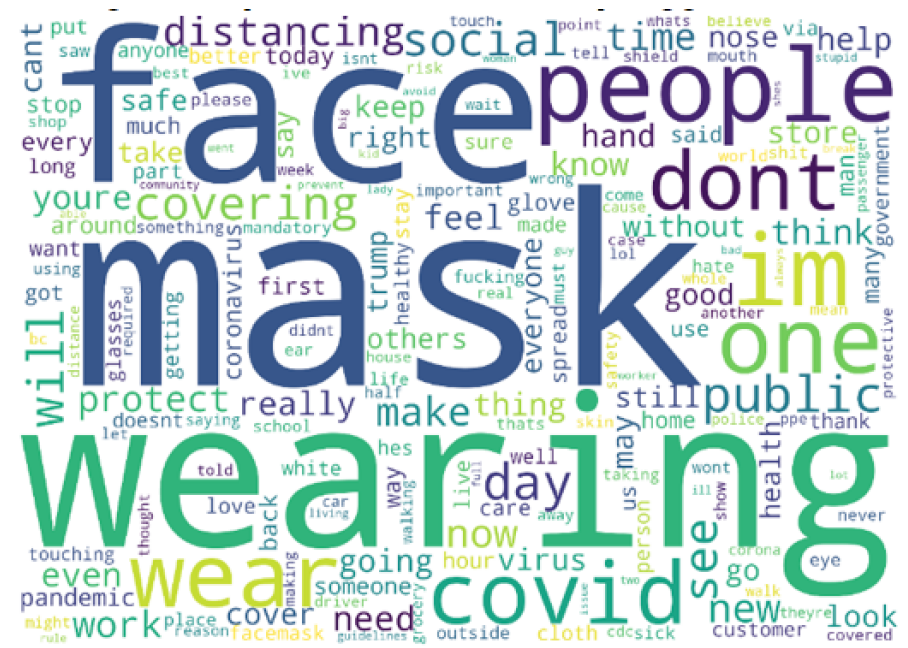

Fig. 2: Word Cloud Generated after pre-processing

words are corrected with pyspellchecker library [20]. Finally, stop words were filtered from the tweets as shown in Figure 2.

After the pre-processing step, tweets are labeled by applying two classes as positive or negative based on the tweet posted by the user whether they support the mask wearing practices. 1200 tweets are labeled as both positive and negative sentiments as shown in Table 1 which avoids the issue of data imbalance by labeling an equal amount of tweets for each classes.

\begin{tabular}{|l|l|}
\hline Sentiment & Labeled Tweets \\
\hline Positive & 600 \\
\hline Negative & 600 \\
\hline Total & 1200 \\
\hline
\end{tabular}

TABLE 1: Labeled sentiments

\subsection{Feature Extraction \& Feature Representation}

The labeled data is transformed into vectors by applying two feature extraction strategies: i) BOW Representation, and ii) Ngram words representation to apply the sparse data with bigram trigram and hybridgram sequence of words. Scikitlearn provides several vectorizer objects to perform feature extraction strategies efficiently and this study uses four of those vectorizers as,

1) CountVectorizer: It's one of the basic and simple ways to tokenize a collection of texts into building a known word. It is used to count the number of times a token shows up in the document and uses this value as its weight [21].

2) TF-IDF Vectorizer: TF-IDF (Term frequency-inverse document frequency) is used to highlight the words which are more significant to the document in a collection of documents. IDF (Inverse Document Frequency) calculates the importance of a term. Some words which may appear in every document such as 'this', 'the', 'what' and 'if' rank lower because they do not add much significance to the document [22].

3) TF: It is a feature representation technique used to describe how often a term occurs in a document. Terms are known to be words, phrases or tokens in a text [23].

4) BR: The binary feature value representation reflects on (i.e., 1 for presence and 0 for absence) features disregarding the occurrence of the term in the document [24].

\subsection{Feature Selection}

This study then applied several Feature selection Techniques to gather informative features by excluding non-informative features from the sparse feature vector which helps to lessen the feature size. Hence, a large number of features may produce less accurate models and tend to increase computational time and complexity [25]. In this study, three main feature selection techniques are applied to compare and contrast the performance of each decision models. The feature selection techniques are as follows.

1) Chi-Squared Test: It is a statistical test used to determine whether there is a statistically important difference between expected and observed frequencies in the feature vector [25][26].

2) Information Gain: It is an entropy-based feature selection method and it is calculated by the use of the term for classification of information by the importance given in a feature vector [27].

3) ANOVA F-Value: Analysis of Variance (ANOVA) is a statistical way to check the means of two or more different groups in a feature vector. This helps to decrease the features by selecting the important features which significantly improve the computation time and accuracy of decision models [28].

\subsection{Decision Models}

There are numerous supervised machine learning decision models available for sentiment analysis [29]. However, each decision model has its unique ways of the learning process. Therefore, picking the most 
applicable decision model for the study is critical. This study mainly uses five decision models, i.e., linear and polynomial SVM kernels, NB, MaxEnt, RF and KNN applied on master feature vectors to find the best performing decision models.

\subsection{Creating Master Feature Vectors}

The tweets are pre-processed via the support of Natural Language Tool Kit (NLTK) [30]. After preprocessing the tweets, features are extracted using BOW, N-Grams as bigram, trigram and hybrid-grams (BOW + bigram + trigram). Then, the study advances to the next step by implementing the feature representation by applying BR, TF, TF-IDF and CountVectorizer. Moreover, best 1000 features are filtered using Chi-Squared Test, information gain, and Anova FValue from the feature vector. Hence, based on the feature extraction, feature representation and feature selection criteria, 48 master feature vector labels are formed as shown in Table 2.

\subsection{Applying Word Embedding Techniques}

This study applies two word embedding techniques apart from master feature vectors. Whereas, Word2Vec and GloVe allow to show the words in vector form (embedding). Hence, Word2vec embedding is based on training a shallow feed-forward neural network, while GloVe embedding is learnt via matrix factorization techniques [31][32].

\section{Results}

The experiments show various findings related to each specified portion mentioned in the methodology. The Train and Test accuracy along with macro precision, macro recall and macro F-measure represented with word embedding techniques, master feature vectors indicating 288 analysis with 4 feature extraction, and 4 feature representation accompanying 5 classification algorithms are shown in Table 3-12. The values which are represented in a table with the lowest are highlighted in bold red and the highest with bold green. Moreover, when comparing to the accuracy of train and test for master feature vectors, lowest training accuracy (0.506), test accuracy $(0.467)$ and the highest training accuracy (0.99), and test accuracy (0.842) were obtained. However, in training sets, all algorithms comparatively perform better except KNN which struggles with information gain as the feature selection scheme. Moreover, for word embedding techniques, the lowest train accuracy (0.65), test accuracy (0.581) and the highest training accuracy (1.0), test accuracy (0.653) are achieved. Therefore, both GloVe and Word2Vec embedding have the highest accuracy when training with NB algorithm.

The performance measurement of master feature Vectors for the lowest macro precision (0.481), macro recall (0.483) and macro F-measure (0.347) were achieved. Whereas, the highest macro precision (0.863), macro recall (0.837) and macro F-measure (0.838) were accomplished by NB algorithm. Overall NB has performed better compared to other five algorithms. In word embedding techniques, lowest macro precision (0.578), macro recall (0.578) and macro Fmeasure (0.578) were obtained by NB algorithm, and the highest macro precision (0.652), macro re-call (0.653) and macro F-measure (0.652) were achieved by linear SVM algorithm.

\section{Discussion}

This study indicates that hybrid-grams and BOW perform well compared to bigram or trigram when applying Chi-squared test, information gain and Anova FValue as feature representation. Whereas, NB decision model performs significantly better compared to other algorithms. Moreover, next to NB algorithm, MaxEnt performs better in all feature extraction, feature selection, and feature representation criteria mentioned in the study. Although, it does not perform well in trigram as feature extraction.

When assessing the Chi-squared test, information gain and Anova F-Value, Chi-squared test gives better performance than information gain and Anova FValue. In terms of feature representation, CountVectorizer comparatively performs well along with other feature representation schemes. This study shows that KNN algorithm does not perform well in all three feature selection techniques compared to other algorithms. Hence, this study finds that the highest macro F-measure and macro precision with feature extraction as hybrid-grams, feature representation as TF-IDF, feature selection as Chi-squared test and the highest macro recall is achieved with feature extraction as BOW, and feature representation as TF-IDF. Feature selection as ANOVA F-value performs better when applying NB as decision model.

This study also uses word embedding techniques to assess the performance of the algorithms. Whereas, linear SVM performs well compared to other algorithms. Although, the highest macro precision, macro recall and macro F-measure values are very low compared to feature vector representation schemes. NB algorithm has the lowest accuracy in both GloVe and Word2Vec word embedding techniques as shown in Figure 3-5 respectively. 


\begin{tabular}{|c|c|c|c|}
\hline Label & Master feature vector & Label & Master feature vector \\
\hline $\mathbf{L 1}$ & $\mathrm{BOW}+\mathrm{BR}+$ Information Gain & $\mathbf{L 2 5}$ & Trigram + BR + Chi-Squared Test \\
\hline L2 & $\mathrm{BOW}+\mathrm{TF}+$ Information Gain & L26 & Trigram + TF + Chi-Squared Test \\
\hline L3 & $\mathrm{BOW}+\mathrm{TF}-\mathrm{IDF}+$ Information Gain & L27 & Trigram + TF-IDF + Chi-Squared Test \\
\hline L4 & BOW + CountVectorizer+ Information Gain & $\mathbf{L 2 8}$ & Trigram + CountVectorizer + Chi-Squared Test \\
\hline L5 & Bigram + BR + Information Gain & $\mathbf{L 2 9}$ & Hybrid-grams + BR + Chi-Squared Test \\
\hline L6 & Bigram + TF + Information Gain & L30 & Hybrid-grams $+\mathrm{TF}+$ Chi-Squared Test \\
\hline L7 & Bigram + TF-IDF + Information Gain & L31 & Hybrid-grams + TF-IDF + Chi-Squared Test \\
\hline L8 & Bigram + CountVectorizer+ Information Gain & L32 & Hybrid-grams + CountVectorizer + Chi-Squared Test \\
\hline L9 & Trigram + BR + Information Gain & L33 & $\mathrm{BOW}+\mathrm{BR}+\mathrm{ANOVA} \mathrm{F-value}$ \\
\hline L10 & Trigram $+\mathrm{TF}+$ Information Gain & L34 & $\mathrm{BOW}+\mathrm{TF}+\mathrm{ANOVA}$ F-value \\
\hline L11 & Trigram + TF-IDF + Information Gain & L35 & $\mathrm{BOW}+\mathrm{TF}$-IDF + ANOVA F-value \\
\hline L12 & Trigram + CountVectorizer + Information Gain & L36 & $\mathrm{BOW}+$ CountVectorizer + ANOVA F-value \\
\hline L13 & Hybrid-grams + BR + Information Gain & L37 & Bigram + BR + ANOVA F-value \\
\hline L14 & Hybrid-grams $+\mathrm{TF}+$ Information Gain & L38 & Bigram + TF + ANOVA F-value \\
\hline L15 & Hybrid-grams + TF-IDF + Information Gain & L39 & Bigram + TF-IDF + ANOVA F-value \\
\hline L16 & Hybrid-grams + CountVectorizer + Information Gain & $\mathbf{L} 40$ & Bigram + CountVectorizer + ANOVA F-value \\
\hline L17 & $\mathrm{BOW}+\mathrm{BR}+$ Chi-Squared Test & L41 & Trigram + BR + ANOVA F-value \\
\hline L18 & $\mathrm{BOW}+\mathrm{TF}+$ Chi-Squared Test & $\mathbf{L} 42$ & Trigram $+\mathrm{TF}+\mathrm{ANOVA}$ F-value \\
\hline L19 & $\mathrm{BOW}+\mathrm{TF}-\mathrm{IDF}+$ Chi-Squared Test & L43 & Trigram + TF-IDF + ANOVA F-value \\
\hline L20 & BOW + CountVectorizer+ Chi-Squared Test & L44 & Trigram + CountVectorizer+ ANOVA F-value \\
\hline L21 & Bigram + BR + Chi-Squared Test & L45 & Hybrid-grams + BR + ANOVA F-value \\
\hline L22 & Bigram $+\mathrm{TF}+$ Chi-Squared Test & L46 & Hybrid-grams $+\mathrm{TF}+$ ANOVA F-value \\
\hline L23 & Bigram + TF-IDF + Chi-Squared Test & $\mathbf{L} 47$ & Hybrid-grams + TF-IDF + ANOVA F-value \\
\hline $\mathrm{L24}$ & Bigram + CountVectorizer+ Chi-Squared Test & L48 & Hybrid-grams + CountVectorizer + ANOVA F-value \\
\hline
\end{tabular}

TABLE 2: Master feature vectors

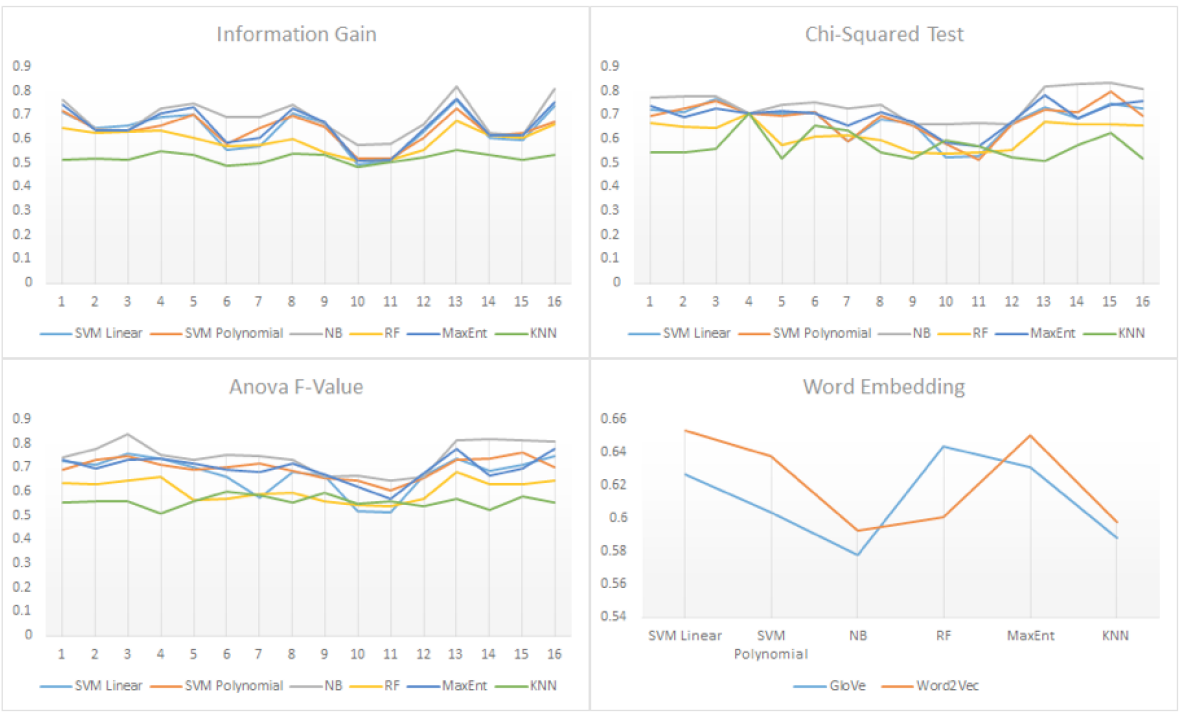

Fig. 3: Macro recall of 288 analysis + word embedding

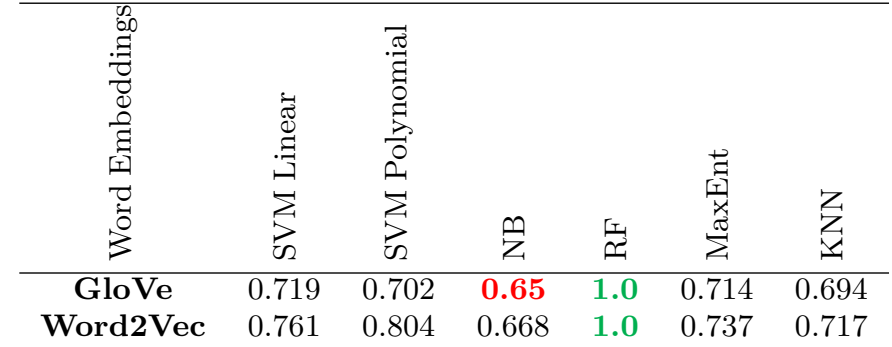

TABLE 3: Train accuracy of word embeddings

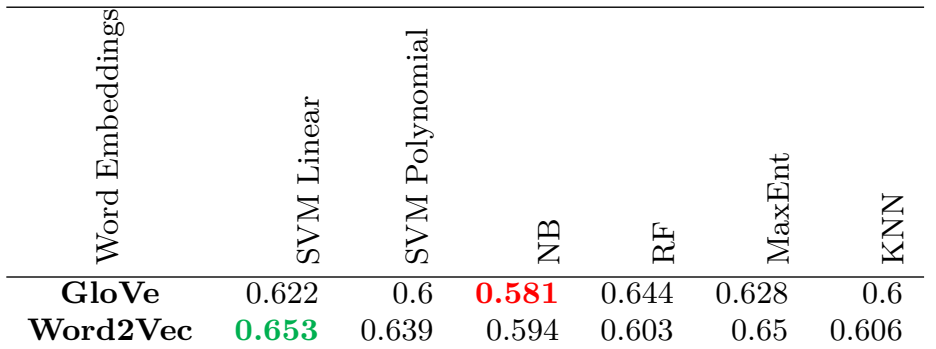

TABLE 4: Test accuracy of word embeddings 

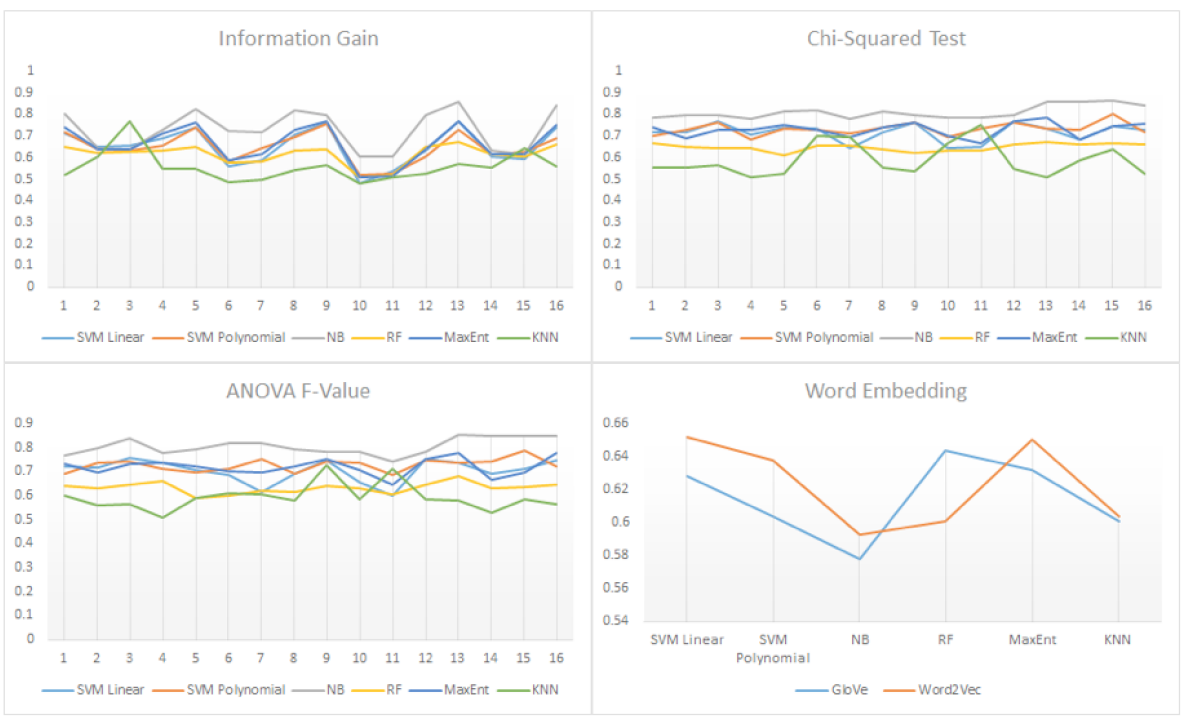

Fig. 4: Macro precision of 288 analysis + word embedding
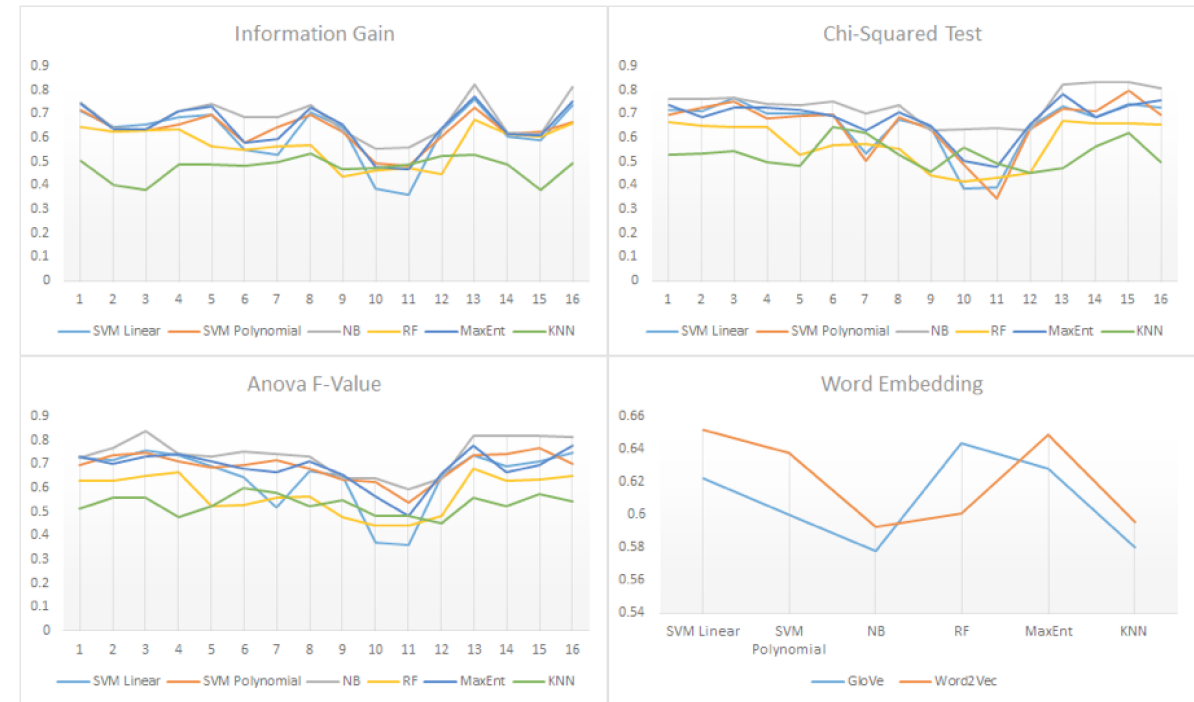

Fig. 5: Macro F-measure of 288 analysis + word embedding

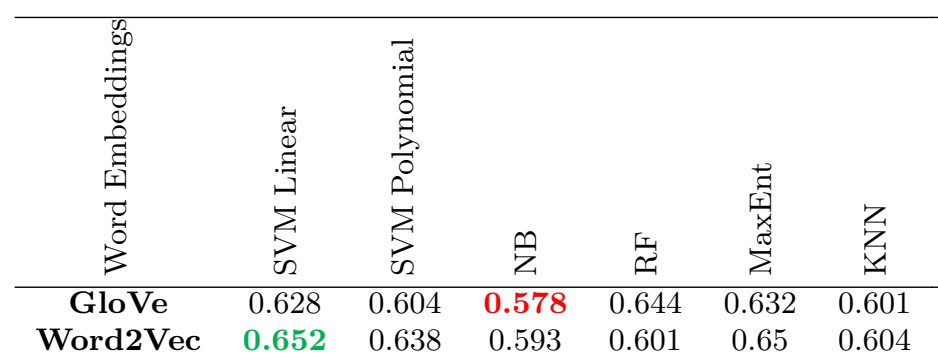

TABLE 5: Macro precision of word embeddings

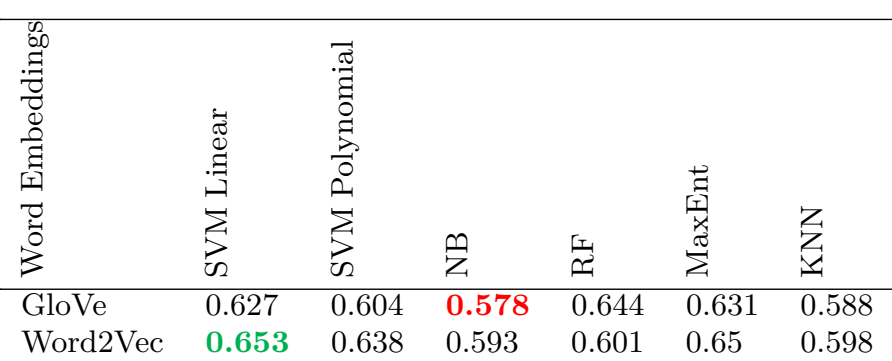

TABLE 6: Macro recall of word embeddings

\section{Conclusion}

This study evaluates the mask-wearing practices during the Covid-19 pandemic via analyzing Twitter tweets to find the performance of several decision models. This study helps to detect whether the maskwearing practices have been implemented or supported. Hence, those who discourage mask-wearing practices can be prevented based on the sentiment 


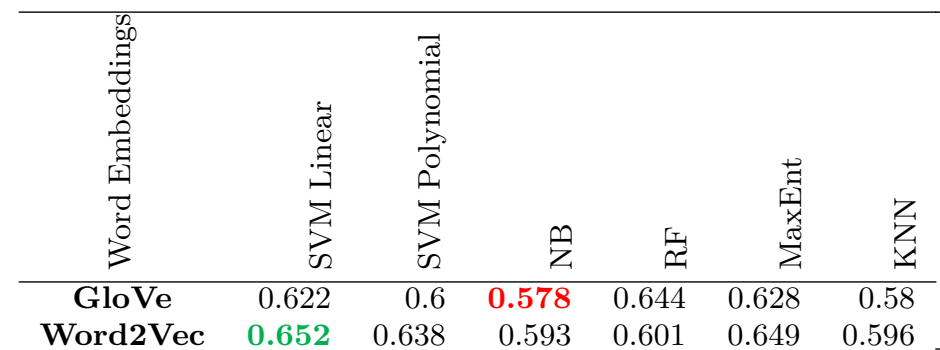

TABLE 7: Macro F-measure of all word embeddings

of the tweets. This study has assessed each decision model's performance with 228 master feature vectors by applying several feature extractions, feature representations, and feature selection schemes. Hence, this study concludes that NB performs significantly better, and KNN has the lowest performance compared to other decision models mentioned in the study. This study has two major limitations: i) increasing the number of labeled tweets, ii) applying more classes to the tweets. As the future direction, we can further enhance the study by applying more supervised machine learning algorithms along with different feature representations and feature selection techniques.

\section{References}

[1] N. Chen, M. Zhou, X. Dong, J. Qu, F. Gong, Y. Han, et al., "Epidemiological and clinical characteristics of 99 cases of 2019 novel coronavirus pneumonia in Wuhan, China: a descriptive study," The Lancet, vol. 395, pp. 507-513, 2020.

[2] X. Chen, L. Ran, Q. Liu, Q. Hu, X. Du, and X. Tan, "Hand Hygiene, Mask-Wearing Behaviors and Its Associated Factors during the COVID-19 Epidemic: A Cross-Sectional Study among Primary School Students in Wuhan, China," International journal of environmental research and public health, vol. 17, p. 2893, 2020.

[3] A. E. Aiello, V. Perez, R. M. Coulborn, B. M. Davis, M. Uddin, and A. S. Monto, "Facemasks, hand hygiene, and influenza among young adults: a randomized intervention trial," PloS one, vol. 7, p. e29744, 2012.

[4] T. Greenhalgh, M. B. Schmid, T. Czypionka, D. Bassler, and L. Gruer, "Face masks for the public during the covid19 crisis," Bmj, vol. 369, 2020.

[5] S. E. Eikenberry, M. Mancuso, E. Iboi, T. Phan, K. Eikenberry, Y. Kuang, et al., "To mask or not to mask: Modeling the potential for face mask use by the general public to curtail the COVID-19 pandemic," Infectious Disease Modelling, 2020.

[6] J. Wu, F. Xu, W. Zhou, D. R. Feikin, C.-Y. Lin, X. He, et al., "Risk factors for SARS among persons without known contact with SARS patients, Beijing, China," Emerging infectious diseases, vol. 10, p. 210, 2004.

[7] S. S. Prasad, J. Kumar, D. K. Prabhakar, and S. Tripathi, "Sentiment mining: An approach for Bengali and Tamil tweets," in 2016 Ninth International Conference on Contemporary Computing (IC3), pp. 1-4, 2016.

\begin{tabular}{|c|c|c|c|c|c|c|}
\hline 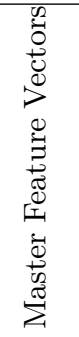 & 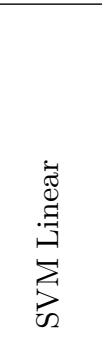 & 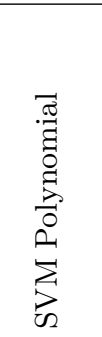 & $\frac{\varphi}{z}$ & 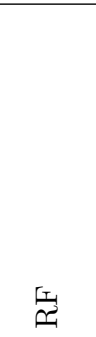 & 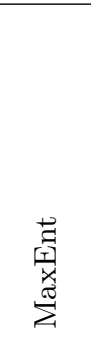 & 忩 \\
\hline L1 & 0.927 & 0.889 & 0.861 & 0.831 & 0.906 & 0.676 \\
\hline L2 & 0.827 & 0.956 & 0.887 & 0.876 & 0.794 & 0.536 \\
\hline L3 & 0.865 & 0.99 & 0.895 & 0.887 & 0.835 & 0.506 \\
\hline L4 & 0.938 & 0.913 & 0.854 & 0.86 & 0.914 & 0.606 \\
\hline L5 & 0.863 & 0.865 & 0.84 & 0.736 & 0.854 & 0.627 \\
\hline L6 & 0.757 & 0.911 & 0.829 & 0.763 & 0.777 & 0.671 \\
\hline L7 & 0.76 & 0.92 & 0.832 & 0.756 & 0.804 & 0.687 \\
\hline L8 & 0.849 & 0.849 & 0.838 & 0.731 & 0.838 & 0.675 \\
\hline L9 & 0.811 & 0.814 & 0.805 & 0.67 & 0.806 & 0.751 \\
\hline L10 & 0.621 & 0.799 & 0.796 & 0.714 & 0.758 & 0.681 \\
\hline L11 & 0.579 & 0.827 & 0.795 & 0.729 & 0.757 & 0.683 \\
\hline L12 & 0.796 & 0.8 & 0.804 & 0.694 & 0.792 & 0.623 \\
\hline L13 & 0.92 & 0.875 & 0.858 & 0.815 & 0.899 & 0.683 \\
\hline L14 & 0.775 & 0.94 & 0.821 & 0.887 & 0.761 & 0.648 \\
\hline L15 & 0.77 & 0.988 & 0.826 & 0.876 & 0.776 & 0.537 \\
\hline L16 & 0.921 & 0.863 & 0.851 & 0.827 & 0.901 & 0.649 \\
\hline L17 & 0.927 & 0.94 & 0.874 & 0.842 & 0.894 & 0.681 \\
\hline L18 & 0.821 & 0.93 & 0.889 & 0.845 & 0.794 & 0.698 \\
\hline L19 & 0.867 & 0.951 & 0.892 & 0.819 & 0.843 & 0.726 \\
\hline L20 & 0.921 & 0.936 & 0.865 & 0.833 & 0.9 & 0.686 \\
\hline L21 & 0.868 & 0.87 & 0.839 & 0.717 & 0.861 & 0.632 \\
\hline L22 & 0.802 & 0.856 & 0.845 & 0.749 & 0.808 & 0.746 \\
\hline L23 & 0.756 & 0.761 & 0.84 & 0.752 & 0.802 & 0.705 \\
\hline L24 & 0.87 & 0.868 & 0.84 & 0.721 & 0.865 & 0.707 \\
\hline L25 & 0.811 & 0.812 & 0.805 & 0.676 & 0.806 & 0.733 \\
\hline L26 & 0.651 & 0.743 & 0.757 & 0.67 & 0.739 & 0.69 \\
\hline L27 & 0.651 & 0.638 & 0.745 & 0.673 & 0.723 & 0.629 \\
\hline L28 & 0.81 & 0.811 & 0.804 & 0.706 & 0.804 & 0.73 \\
\hline L29 & 0.923 & 0.895 & 0.854 & 0.805 & 0.889 & 0.656 \\
\hline L30 & 0.781 & 0.913 & 0.873 & 0.807 & 0.78 & 0.689 \\
\hline L31 & 0.802 & 0.898 & 0.864 & 0.789 & 0.796 & 0.742 \\
\hline L32 & 0.924 & 0.888 & 0.854 & 0.81 & 0.9 & 0.696 \\
\hline L33 & 0.942 & 0.902 & 0.848 & 0.826 & 0.91 & 0.671 \\
\hline L34 & 0.814 & 0.905 & 0.871 & 0.845 & 0.785 & 0.674 \\
\hline L35 & 0.855 & 0.948 & 0.896 & 0.856 & 0.839 & 0.679 \\
\hline L26 & 0.651 & 0.743 & 0.757 & 0.67 & 0.739 & 0.69 \\
\hline L27 & 0.651 & 0.638 & 0.745 & 0.673 & 0.723 & 0.629 \\
\hline L28 & 0.81 & 0.811 & 0.804 & 0.706 & 0.804 & 0.73 \\
\hline L29 & 0.923 & 0.895 & 0.854 & 0.805 & 0.889 & 0.656 \\
\hline L30 & 0.781 & 0.913 & 0.873 & 0.807 & 0.78 & 0.689 \\
\hline L31 & 0.802 & 0.898 & 0.864 & 0.789 & 0.796 & 0.742 \\
\hline L32 & 0.924 & 0.888 & 0.854 & 0.81 & 0.9 & 0.696 \\
\hline L33 & 0.942 & 0.902 & 0.848 & 0.826 & 0.91 & 0.671 \\
\hline L34 & 0.814 & 0.905 & 0.871 & 0.845 & 0.785 & 0.674 \\
\hline L35 & 0.855 & 0.948 & 0.896 & 0.856 & 0.839 & 0.679 \\
\hline L36 & 0.936 & 0.904 & 0.85 & 0.837 & 0.914 & 0.649 \\
\hline L37 & 0.877 & 0.88 & 0.832 & 0.727 & 0.864 & 0.689 \\
\hline L38 & 0.781 & 0.865 & 0.836 & 0.73 & 0.789 & 0.682 \\
\hline L39 & 0.731 & 0.856 & 0.836 & 0.756 & 0.79 & 0.664 \\
\hline L40 & 0.875 & 0.877 & 0.835 & 0.768 & 0.863 & 0.701 \\
\hline L41 & 0.821 & 0.825 & 0.804 & 0.724 & 0.815 & 0.663 \\
\hline L42 & 0.59 & 0.795 & 0.787 & 0.679 & 0.752 & 0.732 \\
\hline L43 & 0.567 & 0.777 & 0.785 & 0.682 & 0.761 & 0.624 \\
\hline L44 & 0.820 & 0.825 & 0.804 & 0.730 & 0.817 & 0.737 \\
\hline L45 & 0.932 & 0.879 & 0.852 & 0.830 & 0.904 & 0.686 \\
\hline L46 & 0.731 & 0.867 & 0.835 & 0.829 & 0.756 & 0.657 \\
\hline L47 & 0.796 & 0.908 & 0.831 & 0.832 & 0.789 & 0.664 \\
\hline L48 & 0.927 & 0.879 & 0.856 & 0.813 & 0.900 & 0.702 \\
\hline
\end{tabular}

TABLE 8: Train accuracy of 288 master feature vector analysis 


\begin{tabular}{|c|c|c|c|c|c|c|}
\hline 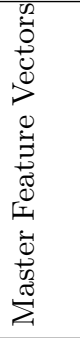 & 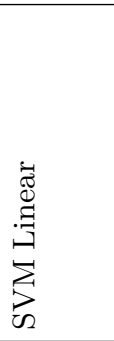 & 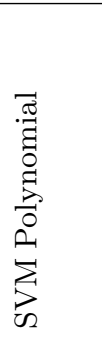 & $\frac{\theta}{z}$ & 崩 & 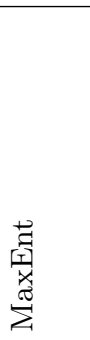 & 学 \\
\hline L1 & 0.714 & 0.719 & 0.75 & 0.65 & 0.742 & 0.528 \\
\hline L2 & 0.65 & 0.642 & 0.644 & 0.625 & 0.636 & 0.547 \\
\hline L3 & 0.658 & 0.631 & 0.639 & 0.628 & 0.636 & 0.547 \\
\hline L4 & 0.689 & 0.656 & 0.717 & 0.633 & 0.711 & 0.575 \\
\hline L5 & 0.714 & 0.714 & 0.761 & 0.586 & 0.742 & 0.517 \\
\hline L6 & 0.55 & 0.581 & 0.703 & 0.558 & 0.581 & 0.497 \\
\hline L7 & 0.55 & 0.647 & 0.7 & 0.567 & 0.597 & 0.506 \\
\hline L8 & 0.706 & 0.697 & 0.758 & 0.586 & 0.728 & 0.547 \\
\hline L9 & 0.686 & 0.672 & 0.683 & 0.517 & 0.692 & 0.514 \\
\hline L10 & 0.467 & 0.506 & 0.594 & 0.489 & 0.494 & 0.494 \\
\hline L11 & 0.475 & 0.506 & 0.594 & 0.497 & 0.494 & 0.519 \\
\hline L12 & 0.628 & 0.606 & 0.683 & 0.525 & 0.639 & 0.528 \\
\hline L13 & 0.767 & 0.731 & 0.831 & 0.675 & 0.772 & 0.569 \\
\hline L14 & 0.606 & 0.617 & 0.631 & 0.617 & 0.614 & 0.553 \\
\hline L15 & 0.589 & 0.628 & 0.619 & 0.606 & 0.611 & 0.544 \\
\hline L16 & 0.739 & 0.681 & 0.819 & 0.664 & 0.753 & 0.556 \\
\hline L17 & 0.719 & 0.703 & 0.767 & 0.667 & 0.739 & 0.558 \\
\hline L18 & 0.717 & 0.728 & 0.767 & 0.65 & 0.689 & 0.558 \\
\hline L19 & 0.769 & 0.75 & 0.769 & 0.647 & 0.728 & 0.55 \\
\hline L20 & 0.703 & 0.683 & 0.747 & 0.647 & 0.728 & 0.519 \\
\hline L21 & 0.719 & 0.708 & 0.758 & 0.558 & 0.731 & 0.536 \\
\hline L22 & 0.703 & 0.703 & 0.767 & 0.592 & 0.697 & 0.672 \\
\hline L23 & 0.569 & 0.564 & 0.711 & 0.597 & 0.642 & 0.656 \\
\hline L24 & 0.694 & 0.708 & 0.758 & 0.578 & 0.722 & 0.558 \\
\hline L25 & 0.686 & 0.678 & 0.683 & 0.517 & 0.689 & 0.5 \\
\hline L26 & 0.494 & 0.553 & 0.683 & 0.508 & 0.561 & 0.617 \\
\hline L27 & 0.497 & 0.481 & 0.686 & 0.514 & 0.542 & 0.6 \\
\hline L28 & 0.686 & 0.681 & 0.683 & 0.528 & 0.692 & 0.503 \\
\hline L29 & 0.736 & 0.731 & 0.831 & 0.672 & 0.783 & 0.525 \\
\hline L30 & 0.686 & 0.719 & 0.839 & 0.664 & 0.686 & 0.586 \\
\hline L31 & 0.742 & 0.8 & 0.842 & 0.667 & 0.736 & 0.633 \\
\hline L32 & 0.728 & 0.708 & 0.817 & 0.661 & 0.758 & 0.536 \\
\hline L33 & 0.725 & 0.694 & 0.728 & 0.631 & 0.733 & 0.578 \\
\hline L34 & 0.717 & 0.736 & 0.769 & 0.631 & 0.697 & 0.564 \\
\hline L35 & 0.758 & 0.744 & 0.839 & 0.647 & 0.731 & 0.558 \\
\hline L36 & 0.736 & 0.708 & 0.742 & 0.664 & 0.739 & 0.525 \\
\hline L37 & 0.692 & 0.683 & 0.747 & 0.547 & 0.708 & 0.578 \\
\hline L38 & 0.65 & 0.694 & 0.767 & 0.553 & 0.681 & 0.611 \\
\hline L39 & 0.553 & 0.728 & 0.761 & 0.575 & 0.669 & 0.6 \\
\hline L40 & 0.672 & 0.678 & 0.747 & 0.578 & 0.708 & 0.572 \\
\hline L41 & 0.686 & 0.675 & 0.683 & 0.536 & 0.689 & 0.622 \\
\hline L42 & 0.489 & 0.667 & 0.686 & 0.517 & 0.6 & 0.528 \\
\hline L43 & 0.481 & 0.581 & 0.625 & 0.514 & 0.542 & 0.589 \\
\hline L44 & 0.686 & 0.678 & 0.683 & 0.542 & 0.692 & 0.514 \\
\hline L45 & 0.739 & 0.736 & 0.825 & 0.683 & 0.778 & 0.581 \\
\hline L46 & 0.692 & 0.742 & 0.825 & 0.631 & 0.667 & 0.522 \\
\hline L47 & 0.711 & 0.772 & 0.822 & 0.636 & 0.692 & 0.572 \\
\hline L48 & 0.747 & 0.711 & 0.819 & 0.65 & 0.778 & 0.567 \\
\hline
\end{tabular}

\begin{tabular}{|c|c|c|c|c|c|c|}
\hline 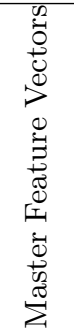 & 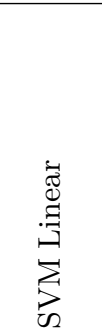 & 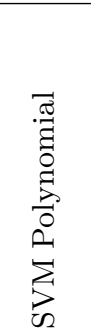 & 皇 & $\frac{I}{\sim \underline{T}}$ & 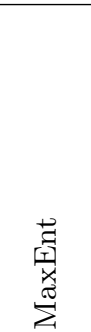 & 学 \\
\hline L1 & 0.713 & 0.719 & 0.8 & 0.648 & 0.741 & 0.518 \\
\hline L2 & 0.648 & 0.64 & 0.643 & 0.623 & 0.636 & 0.604 \\
\hline L3 & 0.657 & 0.63 & 0.637 & 0.629 & 0.637 & 0.77 \\
\hline L4 & 0.689 & 0.654 & 0.728 & 0.634 & 0.709 & 0.55 \\
\hline L5 & 0.738 & 0.742 & 0.822 & 0.647 & 0.762 & 0.549 \\
\hline L6 & 0.557 & 0.582 & 0.725 & 0.578 & 0.587 & 0.488 \\
\hline L7 & 0.587 & 0.646 & 0.715 & 0.582 & 0.614 & 0.498 \\
\hline L8 & 0.705 & 0.696 & 0.82 & 0.631 & 0.728 & 0.541 \\
\hline L9 & 0.762 & 0.758 & 0.797 & 0.636 & 0.766 & 0.564 \\
\hline L10 & 0.481 & 0.52 & 0.606 & 0.509 & 0.51 & 0.481 \\
\hline L11 & 0.535 & 0.527 & 0.603 & 0.518 & 0.516 & 0.506 \\
\hline L12 & 0.63 & 0.606 & 0.797 & 0.648 & 0.641 & 0.526 \\
\hline L13 & 0.766 & 0.731 & 0.858 & 0.674 & 0.771 & 0.571 \\
\hline L14 & 0.603 & 0.616 & 0.63 & 0.617 & 0.614 & 0.553 \\
\hline L15 & 0.595 & 0.626 & 0.617 & 0.604 & 0.615 & 0.645 \\
\hline L16 & 0.738 & 0.692 & 0.843 & 0.662 & 0.753 & 0.558 \\
\hline L17 & 0.719 & 0.703 & 0.788 & 0.665 & 0.738 & 0.554 \\
\hline L18 & 0.716 & 0.727 & 0.797 & 0.649 & 0.688 & 0.553 \\
\hline L19 & 0.768 & 0.762 & 0.794 & 0.645 & 0.727 & 0.565 \\
\hline L20 & 0.705 & 0.682 & 0.781 & 0.646 & 0.727 & 0.51 \\
\hline L21 & 0.742 & 0.733 & 0.816 & 0.612 & 0.751 & 0.525 \\
\hline L22 & 0.733 & 0.731 & 0.817 & 0.654 & 0.729 & 0.698 \\
\hline L23 & 0.642 & 0.712 & 0.778 & 0.656 & 0.694 & 0.693 \\
\hline L24 & 0.718 & 0.74 & 0.816 & 0.636 & 0.741 & 0.554 \\
\hline L25 & 0.762 & 0.762 & 0.797 & 0.62 & 0.764 & 0.535 \\
\hline L26 & 0.646 & 0.695 & 0.783 & 0.632 & 0.701 & 0.666 \\
\hline L27 & 0.652 & 0.737 & 0.785 & 0.632 & 0.667 & 0.75 \\
\hline L28 & 0.767 & 0.763 & 0.797 & 0.66 & 0.766 & 0.546 \\
\hline L29 & 0.736 & 0.737 & 0.858 & 0.671 & 0.783 & 0.51 \\
\hline L30 & 0.685 & 0.727 & 0.861 & 0.662 & 0.685 & 0.586 \\
\hline L31 & 0.745 & 0.802 & 0.863 & 0.667 & 0.744 & 0.636 \\
\hline L32 & 0.727 & 0.719 & 0.844 & 0.66 & 0.758 & 0.526 \\
\hline L33 & 0.724 & 0.693 & 0.769 & 0.64 & 0.732 & 0.6 \\
\hline L34 & 0.716 & 0.735 & 0.798 & 0.63 & 0.696 & 0.56 \\
\hline L35 & 0.757 & 0.744 & 0.839 & 0.646 & 0.73 & 0.562 \\
\hline L36 & 0.736 & 0.71 & 0.778 & 0.662 & 0.738 & 0.51 \\
\hline L37 & 0.709 & 0.697 & 0.794 & 0.59 & 0.723 & 0.592 \\
\hline L38 & 0.685 & 0.712 & 0.821 & 0.602 & 0.703 & 0.612 \\
\hline L39 & 0.615 & 0.751 & 0.818 & 0.621 & 0.696 & 0.603 \\
\hline L40 & 0.689 & 0.691 & 0.794 & 0.617 & 0.723 & 0.58 \\
\hline L41 & 0.748 & 0.744 & 0.783 & 0.64 & 0.75 & 0.726 \\
\hline L42 & 0.655 & 0.738 & 0.785 & 0.628 & 0.708 & 0.587 \\
\hline L43 & 0.6 & 0.688 & 0.741 & 0.604 & 0.646 & 0.712 \\
\hline L44 & 0.753 & 0.747 & 0.783 & 0.646 & 0.752 & 0.585 \\
\hline L45 & 0.738 & 0.736 & 0.854 & 0.682 & 0.777 & 0.58 \\
\hline L46 & 0.691 & 0.742 & 0.849 & 0.629 & 0.667 & 0.527 \\
\hline L47 & 0.71 & 0.788 & 0.848 & 0.634 & 0.694 & 0.584 \\
\hline L48 & 0.746 & 0.722 & 0.851 & 0.648 & 0.777 & 0.563 \\
\hline
\end{tabular}

TABLE 10: Macro precision recall of all 288 master $\begin{array}{ll}\text { TABLE 9: Test accuracy of } 288 \text { master feature vector } & \text { TABLE 10: Macro precis } \\ \text { analysis } & \text { feature vector analysis }\end{array}$ 


\begin{tabular}{|c|c|c|c|c|c|c|}
\hline 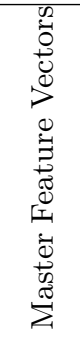 & 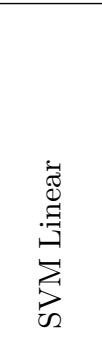 & 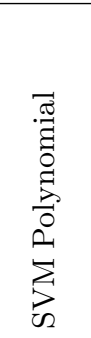 & $\frac{\theta}{z}$ & 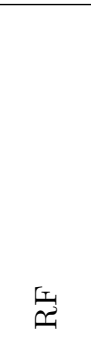 & 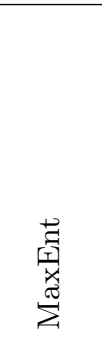 & 学 \\
\hline L1 & 0.714 & 0.716 & 0.763 & 0.647 & 0.74 & 0.516 \\
\hline L2 & 0.646 & 0.64 & 0.643 & 0.624 & 0.636 & 0.517 \\
\hline L3 & 0.657 & 0.631 & 0.636 & 0.629 & 0.637 & 0.515 \\
\hline $\mathrm{L} 4$ & 0.689 & 0.654 & 0.728 & 0.634 & 0.709 & 0.55 \\
\hline L5 & 0.702 & 0.701 & 0.746 & 0.605 & 0.731 & 0.535 \\
\hline L6 & 0.555 & 0.582 & 0.69 & 0.57 & 0.586 & 0.489 \\
\hline L7 & 0.567 & 0.646 & 0.689 & 0.576 & 0.606 & 0.499 \\
\hline L8 & 0.706 & 0.696 & 0.743 & 0.602 & 0.729 & 0.539 \\
\hline L9 & 0.667 & 0.651 & 0.661 & 0.544 & 0.673 & 0.536 \\
\hline L10 & 0.493 & 0.517 & 0.577 & 0.506 & 0.508 & 0.483 \\
\hline L11 & 0.506 & 0.521 & 0.578 & 0.514 & 0.512 & 0.505 \\
\hline L12 & 0.63 & 0.606 & 0.661 & 0.552 & 0.641 & 0.526 \\
\hline L13 & 0.765 & 0.726 & 0.821 & 0.674 & 0.77 & 0.553 \\
\hline L14 & 0.603 & 0.616 & 0.623 & 0.617 & 0.615 & 0.532 \\
\hline L15 & 0.594 & 0.624 & 0.613 & 0.605 & 0.615 & 0.513 \\
\hline L16 & 0.736 & 0.67 & 0.81 & 0.662 & 0.75 & 0.535 \\
\hline L17 & 0.72 & 0.698 & 0.775 & 0.665 & 0.738 & 0.544 \\
\hline L18 & 0.713 & 0.727 & 0.777 & 0.65 & 0.689 & 0.546 \\
\hline L19 & 0.768 & 0.756 & 0.779 & 0.644 & 0.728 & 0.56 \\
\hline L20 & 0.705 & 0.705 & 0.705 & 0.705 & 0.705 & 0.705 \\
\hline L21 & 0.708 & 0.696 & 0.743 & 0.577 & 0.719 & 0.517 \\
\hline L22 & 0.714 & 0.713 & 0.753 & 0.61 & 0.708 & 0.658 \\
\hline L23 & 0.59 & 0.59 & 0.727 & 0.615 & 0.657 & 0.638 \\
\hline L24 & 0.682 & 0.695 & 0.743 & 0.596 & 0.711 & 0.544 \\
\hline L25 & 0.667 & 0.657 & 0.661 & 0.544 & 0.67 & 0.521 \\
\hline L26 & 0.525 & 0.579 & 0.662 & 0.537 & 0.587 & 0.595 \\
\hline L27 & 0.528 & 0.513 & 0.665 & 0.542 & 0.568 & 0.572 \\
\hline L28 & 0.666 & 0.66 & 0.661 & 0.555 & 0.673 & 0.525 \\
\hline L29 & 0.733 & 0.723 & 0.821 & 0.671 & 0.782 & 0.507 \\
\hline L30 & 0.685 & 0.711 & 0.83 & 0.66 & 0.686 & 0.573 \\
\hline L31 & 0.745 & 0.796 & 0.833 & 0.66 & 0.741 & 0.624 \\
\hline L32 & 0.726 & 0.699 & 0.807 & 0.657 & 0.756 & 0.52 \\
\hline L33 & 0.725 & 0.692 & 0.74 & 0.637 & 0.733 & 0.556 \\
\hline L34 & 0.713 & 0.734 & 0.779 & 0.631 & 0.697 & 0.558 \\
\hline L35 & 0.757 & 0.745 & 0.837 & 0.647 & 0.731 & 0.561 \\
\hline L36 & 0.737 & 0.711 & 0.753 & 0.662 & 0.739 & 0.507 \\
\hline L37 & 0.7 & 0.69 & 0.733 & 0.565 & 0.716 & 0.558 \\
\hline L38 & 0.663 & 0.703 & 0.752 & 0.571 & 0.69 & 0.6 \\
\hline L39 & 0.573 & 0.716 & 0.746 & 0.592 & 0.68 & 0.587 \\
\hline L40 & 0.68 & 0.685 & 0.733 & 0.593 & 0.716 & 0.553 \\
\hline L41 & 0.668 & 0.656 & 0.662 & 0.561 & 0.671 & 0.597 \\
\hline L42 & 0.52 & 0.647 & 0.665 & 0.544 & 0.622 & 0.55 \\
\hline L43 & 0.512 & 0.603 & 0.647 & 0.54 & 0.567 & 0.561 \\
\hline L44 & 0.667 & 0.658 & 0.662 & 0.567 & 0.674 & 0.539 \\
\hline L45 & 0.737 & 0.733 & 0.815 & 0.68 & 0.776 & 0.568 \\
\hline L46 & 0.688 & 0.738 & 0.816 & 0.628 & 0.668 & 0.526 \\
\hline L47 & 0.711 & 0.763 & 0.813 & 0.632 & 0.694 & 0.58 \\
\hline L48 & 0.746 & 0.702 & 0.809 & 0.647 & 0.776 & 0.554 \\
\hline
\end{tabular}

\begin{tabular}{|c|c|c|c|c|c|c|}
\hline 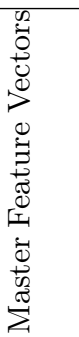 & 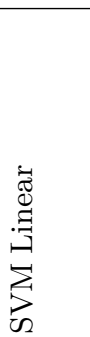 & 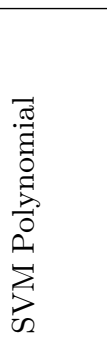 & $\frac{\varphi}{z}$ & $\stackrel{\Gamma}{\sim}$ & 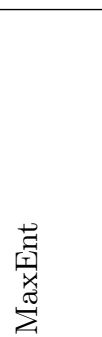 & 忩 \\
\hline L1 & 0.713 & 0.717 & 0.745 & 0.647 & 0.74 & 0.505 \\
\hline L2 & 0.646 & 0.64 & 0.643 & 0.623 & 0.635 & 0.403 \\
\hline L3 & 0.657 & 0.63 & 0.636 & 0.628 & 0.636 & 0.38 \\
\hline L4 & 0.688 & 0.654 & 0.713 & 0.633 & 0.71 & 0.49 \\
\hline L5 & 0.698 & 0.696 & 0.741 & 0.562 & 0.73 & 0.487 \\
\hline L6 & 0.549 & 0.58 & 0.686 & 0.551 & 0.58 & 0.484 \\
\hline L7 & 0.529 & 0.646 & 0.686 & 0.562 & 0.593 & 0.496 \\
\hline L8 & 0.705 & 0.696 & 0.738 & 0.568 & 0.728 & 0.535 \\
\hline L9 & 0.646 & 0.625 & 0.632 & 0.436 & 0.654 & 0.467 \\
\hline L10 & 0.387 & 0.495 & 0.556 & 0.461 & 0.479 & 0.472 \\
\hline L11 & 0.359 & 0.485 & 0.561 & 0.473 & 0.467 & 0.487 \\
\hline L12 & 0.628 & 0.605 & 0.632 & 0.45 & 0.639 & 0.526 \\
\hline L13 & 0.765 & 0.727 & 0.824 & 0.674 & 0.771 & 0.531 \\
\hline L14 & 0.603 & 0.616 & 0.622 & 0.616 & 0.614 & 0.489 \\
\hline L15 & 0.588 & 0.624 & 0.613 & 0.604 & 0.611 & 0.383 \\
\hline L16 & 0.737 & 0.666 & 0.813 & 0.662 & 0.75 & 0.494 \\
\hline L17 & 0.719 & 0.698 & 0.765 & 0.665 & 0.738 & 0.527 \\
\hline L18 & 0.714 & 0.727 & 0.764 & 0.649 & 0.688 & 0.536 \\
\hline L19 & 0.768 & 0.75 & 0.768 & 0.644 & 0.727 & 0.544 \\
\hline L20 & 0.703 & 0.682 & 0.744 & 0.646 & 0.727 & 0.5 \\
\hline L21 & 0.704 & 0.691 & 0.739 & 0.53 & 0.718 & 0.483 \\
\hline L22 & 0.699 & 0.699 & 0.75 & 0.568 & 0.693 & 0.648 \\
\hline L23 & 0.536 & 0.504 & 0.701 & 0.576 & 0.629 & 0.621 \\
\hline L24 & 0.676 & 0.688 & 0.739 & 0.552 & 0.709 & 0.527 \\
\hline L25 & 0.646 & 0.633 & 0.632 & 0.442 & 0.65 & 0.456 \\
\hline L26 & 0.385 & 0.489 & 0.636 & 0.419 & 0.502 & 0.559 \\
\hline L27 & 0.39 & 0.347 & 0.64 & 0.432 & 0.477 & 0.495 \\
\hline L28 & 0.645 & 0.637 & 0.632 & 0.452 & 0.654 & 0.453 \\
\hline L29 & 0.733 & 0.724 & 0.824 & 0.671 & 0.782 & 0.475 \\
\hline L30 & 0.685 & 0.711 & 0.833 & 0.661 & 0.685 & 0.563 \\
\hline L31 & 0.742 & 0.798 & 0.836 & 0.66 & 0.736 & 0.621 \\
\hline L32 & 0.726 & 0.698 & 0.809 & 0.657 & 0.757 & 0.498 \\
\hline L33 & 0.724 & 0.693 & 0.723 & 0.63 & 0.732 & 0.512 \\
\hline L34 & 0.714 & 0.734 & 0.767 & 0.63 & 0.697 & 0.558 \\
\hline L35 & 0.757 & 0.744 & 0.838 & 0.646 & 0.73 & 0.558 \\
\hline L36 & 0.736 & 0.708 & 0.738 & 0.662 & 0.738 & 0.477 \\
\hline L37 & 0.69 & 0.682 & 0.728 & 0.522 & 0.707 & 0.523 \\
\hline L38 & 0.643 & 0.693 & 0.749 & 0.525 & 0.678 & 0.595 \\
\hline L39 & 0.518 & 0.714 & 0.742 & 0.555 & 0.665 & 0.577 \\
\hline L40 & 0.67 & 0.677 & 0.728 & 0.561 & 0.707 & 0.523 \\
\hline L41 & 0.65 & 0.634 & 0.636 & 0.475 & 0.654 & 0.545 \\
\hline L42 & 0.371 & 0.622 & 0.64 & 0.439 & 0.564 & 0.483 \\
\hline L43 & 0.359 & 0.538 & 0.593 & 0.442 & 0.483 & 0.481 \\
\hline L44 & 0.649 & 0.638 & 0.636 & 0.483 & 0.658 & 0.452 \\
\hline L45 & 0.737 & 0.733 & 0.817 & 0.68 & 0.776 & 0.557 \\
\hline L46 & 0.689 & 0.739 & 0.818 & 0.628 & 0.666 & 0.522 \\
\hline L47 & 0.71 & 0.764 & 0.815 & 0.633 & 0.692 & 0.57 \\
\hline L48 & 0.746 & 0.7 & 0.811 & 0.647 & 0.777 & 0.544 \\
\hline
\end{tabular}

TABLE 11: Macro recall of all 288 master feature TABLE 12: Macro F-measure of all 288 master feature vector analysis vector analysis 
[8] R. M. Duwairi, R. Marji, N. Sha'ban, and S. Rushaidat, "Sentiment analysis in Arabic tweets," in 2014 5th International Conference on Information and Communication Systems (ICICS), pp. 1-6, 2014.

[9] C. Troussas, M. Virvou, K. J. Espinosa, K. Llaguno, and J. Caro, "Sentiment analysis of Facebook statuses using Naive Bayes classifier for language learning," in IISA, pp. 1-6, 2013.

[10] G. Gautam and D. Yadav, "Sentiment analysis of twitter data using machine learning approaches and semantic analysis," in 2014 Seventh International Conference on Contemporary Computing (IC3), pp. 437-442, 2014.

[11] S. B. Kotsiantis, I. Zaharakis, and P. Pintelas, "Supervised machine learning: A review of classification techniques," Emerging artificial intelligence applications in computer engineering, vol. 160, pp. 3-24, 2007.

[12] M. Anjaria and R. M. R. Guddeti, "A novel sentiment analysis of social networks using supervised learning," Social Network Analysis and Mining, vol. 4, p. 181, 2014.

[13] L. Ramachandran and E. F. Gehringer, "Automated assessment of review quality using latent semantic analysis," in 2011 IEEE 11th International Conference on Advanced Learning Technologies, pp. 136-138, 2011.

[14] M. Sethi, S. Pandey, P. Trar, and P. Soni, "Sentiment identification in COVID-19 specific tweets," in 2020 International Conference on Electronics and Sustainable Communication Systems (ICESC), pp. 509-516, 2020.

[15] S. C. Eshan and M. S. Hasan, "An application of machine learning to detect abusive bengali text," in 2017 20th International Conference of Computer and Information Technology (ICCIT), pp. 1-6, 2017.

[16] G. Mujtaba, L. Shuib, R. G. Raj, R. Rajandram, and K. Shaikh, "Prediction of cause of death from forensic autopsy reports using text classification techniques: A comparative study," Journal of forensic and legal medicine, vol. 57, pp. 41-50, 2018.

[17] J. Samuel, G. Ali, M. Rahman, E. Esawi, and Y. Samuel, "Covid-19 public sentiment insights and machine learning for tweets classification," Information, vol. 11, p. 314, 2020.

[18] A. Sharma and A. Daniels, "Tweets Sentiment Analysis via Word Embeddings and Machine Learning Techniques," arXiv preprint arXiv:2007.04303, 2020.

[19] Tweepy libray. Available: http://tweepy.github.com/

[20] pyspellchecker. Available: https://pypi.org/project/

[21] CountVectorizer. Available: https://scikit-learn.org

[22] TfidfVectorizer. Available: https://scikitlearn.org/stable/modules

[23] G. Salton and M. J. McGill, "Introduction to Modern Information Retrieval." McGraw-Hill, Inc., 1986.

[24] A. McCallum and K. Nigam, "A comparison of event models for naive bayes text classification," in AAAI-98 workshop on learning for text categorization, pp. 41-48, 1998.

[25] Y. Yang and J. O. Pedersen, "A comparative study on feature selection in text categorization," in Icml, p. 35, 1997.

[26] I. H. Witten and E. Frank, "Data mining: practical machine learning tools and techniques with Java implementations," Acm Sigmod Record, vol. 31, pp. 76-77, 2002.

[27] S. Lei, "A feature selection method based on information gain and genetic algorithm," in 2012 International Conference on Computer Science and Electronics Engineering, pp. 355-358, 2012.

[28] N. O. F. Elssied, O. Ibrahim, and A. H. Osman, "A novel feature selection based on one-way anova F-test for e-mail spam classification," Research Journal of Applied Sciences, Engineering and Technology, vol. 7, pp. 625-638, 2014.
[29] S. B. Kotsiantis, I. D. Zaharakis, and P. E. Pintelas, "Machine learning: a review of classification and combining techniques," Artificial Intelligence Review, vol. 26, pp. 159190, 2006.

[30] S. Bird, E. Klein, and E. Loper, "Natural language processing with Python: analyzing text with the natural language toolkit." " O'Reilly Media, Inc.", 2009.

[31] T. Mikolov, K. Chen, G. Corrado, and J. Dean, "Efficient estimation of word representations in vector space," arXiv preprint arXiv:1301.3781, 2013.

[32] J. Pennington, R. Socher, and C. D. Manning, "Glove: Global vectors for word representation," in Proceedings of the 2014 conference on empirical methods in natural language processing (EMNLP), pp. 1532-1543, 2014. 